\title{
The intestinal microbiota and probiotics in irritable bowel syndrome
}

Jennifer A. J. Madden

MRC Microbiology and Gut Biology Group, University of Dundee, Department Molecular and Cellular Pathology,

Ninewells Hospital and Medical School, Dundee, UK

\section{Abstract}

Irritable bowel syndrome (IBS) is a poorly understood gastrointestinal condition affecting approximately onefifth of the UK population, with a higher prevalence in women and accounting for up to half of referrals to gastroenterology clinics in the UK. It is characterized by abdominal pain, excessive flatus, variable bowel habit and abdominal bloating with no evidence of organic disease. IBS commonly occurs after gastroenteritis or following a course of antibiotics. Suggested aetiologies include motility and psychological disorders and psychophysiological phenomena, although there is also evidence that disruption to the intestinal microbiota can play a role in IBS. Episodes such as hysterectomy, first incidence of bacterial gastroenteritis or a course of antibiotics have been shown to contribute to symptoms compatible with those of IBS. The intestinal microflora in IBS has been shown to differ from that of healthy individuals. Faecal microfloras of IBS patients harbour higher numbers of facultative organisms, such as Klebsiella spp. and enterococci, and low numbers of lactobacilli and bifidobacteria. The role of probiotics in IBS has not been clearly defined. Some studies have shown improvements in pain and flatulence in response to probiotic supplementation, while others have shown no symptomatic or objective improvements. Administration of probiotics reduced caecal and faecal yeast proliferation in IBS patients treated with antibiotics, suggesting that they prevent the disruption of the intestinal microbiota known to be associated with IBS. It is possible that the role of probiotics in the management of IBS will lie in prevention, rather than cure.

Keywords: IBS; microflora; probiotics

Received: 12 Jan. 2004; Accepted: 21 Jan. 2004

\section{Introduction}

Irritable bowel syndrome (IBS) is a frequent and often debilitating bowel disorder in which patients commonly present with bloating and abdominal pain. It can be difficult to diagnose and may require extensive diagnostic testing that is further hindered by the lack of a positive biological or pathogenic marker. This renders the diagnosis of IBS as being one of exclusion of other diseases (1). The Rome II (Table 1) and Manning Criteria provide comprehensive diagnostic guides for practitioners but, because IBS is a multifactorial condition, they do not include all of the clinical characteristics (3). The cause of IBS is unknown: postulated causes include malfermentation of food residues, increased visceral hypersensitivity, psychosocial factors and altered gastrointestinal motility, but it may also be possible that IBS encompasses several or all of these factors. It is thought that IBS affects in the region of onefifth of the UK population at some stage in their lives, although $60-75 \%$ symptomatic people do not visit their general practitioner (4). This may well be related to the severity of their symptoms, since IBS can be a chronic condition in which symptom-free periods and relapses occur (5).

Interest in the use of probiotics in IBS stems from the possible role of the intestinal microbiota in its aetiology. The composition of the gut microbiota is known to be relatively stable in healthy individuals, in that certain bacterial species can be consistently detected in samples collected from the gastrointestinal tract (6). Stability of the microbiota is established by several mechanisms including gastric acidity, gut mobility, bile, products of immune cells in the gut epithelium, competition between microorganisms for nutrients and availability of intestinal binding sites (7). In certain circumstances, this equilibrium can become unbalanced through diet, geographical location, pathological conditions, surgery to the gastrointestinal tract and, most signifi- 
Table I. The Modified Rome Diagnostic Criteria for irritable bowel syndrome (2)

At least 6 months of recurrent symptoms of abdominal pain/discomfort which is:
relieved with defecation
and/or associated with a change in stool frequency
and/or associated with a change in stool consistency
AND
Two or more of:
altered stool frequency
altered stool form
altered stool passage
passage of mucus
bloating or feeling of abdominal distension

at least $25 \%$ of the time

cantly, drug ingestion, particularly antimicrobials (8).

There are links between disruption to the intestinal microbiota and the onset of IBS. Forty per cent of patients surveyed in a retrospective study credited the onset of their IBS to a specific occasion, such as a course of antibiotics, abdominal or pelvic surgery, or a bout of gastroenteritis (9), while significantly more women with IBS reported having previously undergone a hysterectomy than non-sufferers [odds ratio (OR) 1.6, $p<0.01$ ] (10). Gastroenteritis can also alter the gastrointestinal microbiota and its role in the onset of IBS has been comprehensively studied. Of 75 patients questioned who were hospitalized with acute gastroenteritis, 22 had IBScompatible symptoms after the initial illness, with 20 patients still suffering persistent symptoms after 6 months (11). Similarly, a cohort study (12) investigated 303 people with a first incidence of bacterial gastroenteritis and compared them with a control cohort of 575169 people from the general practice research database. Subjects were followed up after 1 year. Of those in the control group, 2027 developed IBS (incidence per 1000 persons per year was 3.4), in contrast to 12 people in the study group (incidence per 1000 persons per year was 39.7, Relative Risk [RR] 11.9), showing strong evidence for the development of IBS following acute bacterial infections. Current data substantiate the role of bacterial infection in the aetiology to the extent that the term postinfectious IBS (PI-IBS) has been devised to describe this subgroup of patients (13).

The indigenous microbiota takes part in many physiological and pathophysiological reactions, and may influence the metabolic activities of certain drugs, all of which can be profoundly influenced by antibiotic therapy. The amount of change caused by an antimicrobial agent is dependent on various factors, including the antimicrobial activity spectrum, pharmokinetics, dose, route of administration and concentration reached in the intestine (14-17). Disruption to the normal microbiota is usually through incomplete absorption of orally administered antibiotics, as well as secretion of the antimicrobial by the salivary glands, in the bile or from the intestine (15). Two prospective studies have investigated the role of antibiotics in the aetiology of IBS. Three-hundred patients undergoing hysterectomy, who were given either prophylactic metronidazole or placebo, were studied in a double-blind, controlled trial. IBS-type symptoms were reported in a greater number of patients undergoing antibiotic prophylaxis than those on placebo (16). In a more recent study, Mendall and Kumar (17) investigated 421 patients attending a general practice clinic. The Manning Criteria were used to assess symptoms and 48 of the subjects screened were symptomatic of having IBS, which was strongly associated with the use of antibiotics (OR 3.7).

\section{The gastrointestinal microbiota in irritable bowel syndrome}

While there is substantial evidence to indicate that events leading to disturbances in the gastrointestinal microbiota may be involved in the aetiology of IBS, there is also some evidence that the microflora of patients with IBS differs from that of healthy individuals. The faecal microbiotas of 20 patients with IBS were investigated using conventional microbiological techniques, and compared with those of 20 healthy controls (18). The microbiota of IBS patients had significantly lower numbers of Enterobacteriaceae, lactobacilli and bifidobacteria compared with controls. It was concluded that, while the microbiota of IBS and healthy subjects were qualitatively very similar, there were quantitative differences that might be of aetiological importance. Bradley et al. (19) found considerable variation in total bacterial counts over an 18 month period in the faecal microbiota of a patient suffering from food-related IBS, with a high proportion of facultative anaerobic species. Similarly, Wyatt et al. (20) investigated the faecal microbiotas of two patients with food-related IBS, before and after challenges with foods thought to provoke symptoms. Few changes were observed in the major bacterial species during these challenges, although 
in one patient levels of lactobacilli and bifidobacteria increased from 21 to $43 \%$ of the total microbiota.

The caecal biopsy-associated, caecal luminal and faecal microbiotas of six patients fulfilling the Rome II Criteria for IBS were compared with those of six controls. A $100 \mathrm{ml}$ phosphate enema was used to clean the left side of the bowel, leaving the right side, including the caecum, undisturbed. To maintain an anaerobic environment carbon dioxide, rather than oxygen, was used to insufflate the bowel. Significantly higher numbers of anaerobes were isolated from the stools of healthy individuals, compared with IBS patients. In the IBS patients, lactobacilli were detected in caecal biopsies and caecal lumen, but not in the faeces. Facultative anaerobes were detected in the caecal biopsies of five IBS patients compared with two healthy subjects (21).

There is therefore some evidence that the gut microbiota of IBS patients differs from that of healthy individuals, although these studies were undertaken using small samples of patients, and larger trials should be conducted to confirm these results, or the studies should be repeated with a defined subset of patients, e.g. diarrhoea-predominant patients. It is also unclear as to whether alterations in the microbiota seen in IBS patients are a cause of IBS, or a result of the disturbed gut motility induced by the disease.

\section{Probiotics and irritable bowel syndrome}

Fermented milks and milk products have been in use since antiquity; however, it was the Russian Nobel laureate Eli Metchkinoff who proposed in 1907 that the longevity of Balkan peoples could be attributed to their ingestion. Probiotics are live microbial food supplements that change either the composition or metabolic activities of the microbiota, or modulate immune system reactivity in a way that benefits health (22). Lactobacilli are the most common species used for this purpose, although bifidobacteria, streptococci and saccharomyces have also been used (23) (Table 2). Their therapeutic effects may result from competitive interactions with the commensal and pathogenic microbiotas, and their influence on the host immune system (26).

IBS patients are not a homogeneous group to study and, as such, few studies have been undertaken to investigate the role of probiotics in this
Table 2. Organisms used as probiotics in humans and animals

\begin{tabular}{lll} 
Lactobacillus species & Bifidobacterium species & Other organisms \\
\hline L. acidophilus & B. adolescentis & Enterococcus faecalis \\
L. casei & B. animalis & Enterococcus faecium \\
L. crispatus & B. bifidum & Lactococcus lactis \\
L. delbrueckii & B. breve & Leuconostoc mesenteroides \\
L. gallinarum & B. infantis & Pediococcus acidilactici \\
L. gasseri & B. longum & Pediococcus pentosaceus \\
L. johnsonii & & Saccharomyces boulardii \\
L. paracasei & & Sporolactobacillus inulinus \\
L. plantarum & & Streptococcus thermophilus \\
L. reuteri & & \\
\hline
\end{tabular}

Adapted from Refs (24) and (25).

disease. Some investigations, using symptomatic differences as a definable endpoint, have shown significant improvements following probiotics (2729 ), although others are subjective and likely to contain a certain amount of placebo response $(30,31)$.

Lactobacillus rhamnosus strain GG (LGG) was given to 24 patients with typical symptoms of IBS in a randomized, double-blind cross-over trial. Of the 19 patients who completed the trial, no differences in pain, urgency and bloating occurred between those given probiotics and those who received the placebo, although there was a reduction in diarrhoea in the LGG group (32). The authors suggested that, although the probiotic had little effect on IBS symptoms, they may have a role in the treatment of diarrhoea in a subset of patients. A recent feeding study investigated the role of probiotics in diarrhoea-predominant IBS patients, in which $3 \mathrm{~g} \mathrm{day}^{-1}$ of $3 \times 10^{11} \mathrm{~g}^{-1}$ VSL\#3 was given to 42 patients with diarrhoea-predominant IBS, for a period of 20 days (33). VSL\#3 contains four strains of lactobacilli, three strains of bifidobacteria and Streptococcus salivarius subsp. thermophilus. Foods that patients had excluded following the diarrhoea episode were reintroduced during the time of probiotic feeding to attempt to reintroduce a normal balanced diet. Following treatment, only eight patients $(19 \%)$ were still symptomatic, while $81 \%$ reported the disappearance of symptoms. The average number of daily bowel movements decreased significantly from 7.2 to $2.1 \quad(p<0.002)$. At the end of probiotic treatment, 32 patients had returned to, and were tolerating, a normal and balanced diet. Stool specimens were only obtained from 10 patients but still showed increases in 
lactobacilli, bifidobacteria and $S$. thermophilus following feeding, while $\beta$-D-galactosidase levels increased significantly $(p<0.01)$. Although this was an unblended and uncontrolled study, the results still show trends towards the beneficial effects of probiotics in the treatment of diarrhoea associated with IBS. Probiotics have had good success in the treatment of antibiotic-associated diarrhoea (34-38); further studies in this subgroup of patients appear to be warranted.

When Lactobacillus plantarum $299 \mathrm{~V}$ was given to 12 patients fulfilling the Rome Criteria for IBS, a reduction in breath hydrogen following ingestion of lactulose occurred, but no reduction in total hydrogen production or any symptomatic improvement was evident (39). It is possible that the dosage $\left(6.25 \times 10^{9} \mathrm{cfu} \mathrm{day}^{-1}\right)$ was too low to have any positive impact. The effect of a combination of $B$. longum and $L$. acidophilus at $2.5 \times 10^{10} \mathrm{cfu}^{\mathrm{day}}{ }^{-1}$ on the caecal and faecal microbiota of IBS patients being treated with antibiotics was investigated (40). The combination of metronidazole and amoxicillin increased numbers of Candida albicans associated with caecal biopsies, the caecal lumen and the faeces in five out of six patients recruited, and yeasts were detected in the stools of three of these patients 2 weeks after cessation of treatment. Of the six patients given antibiotic supplemented with probiotics, C. albicans was isolated from just one patient following treatment, and was not recovered from stool samples provided by patients at day 28 . This suggests a trend towards a preventive, rather than a therapeutic, role for probiotics in the management of IBS; a larger study is warranted to elucidate these results.

\section{Conclusion}

The intestinal microbiota of IBS patients has been extensively studied by conventional microbiological means and differs from that of healthy individuals. There is increasing scientific evidence supporting the rationale of using probiotics in IBS, and it may be that probiotics will have a dual role to play in both the prevention of IBS and the treatment of its associated symptoms.

\section{References}

1. Maxwell PR, Mendall MA, Kumar D. Irritable bowel syndrome. Lancet 1997; 350: 1691-5.

2. Thompson WG, Longstreth GF, Drossman DA, Heaton KW, Irvine EJ, Muller-Lissner SA. Functional bowel disorders and functional abdominal pain. Gut 1999; 45(Suppl 2): I43-7.

3. Camilleri M. Management of the irritable bowel syndrome. Gastroenterology 2001; 120: 652-68.

4. Farthing M. Fortnightly review: Irritable bowel, irritable body, or irritable brain? Br Med J 1995; 310: 171-5.

5. van der Horst HE, van Dulmen AM, Schellevis FG, van Eijk JTM, Fennis JFM, Bleijenberg G. Do patients with irritable bowel syndrome in primary care really differ from outpatients with irritable bowel syndrome? Gut 1997; 41: 669-74.

6. Savage DC. Microbial ecology of the gastrointestinal tract. Annu Rev Microbiol 1977; 31: 107-33.

7. Marshall JC. Gastrointestinal flora and its alterations in critical illness. Curr Opin Nutr Metab Care 1999; 2: 40511.

8. Neilsen $\mathrm{OH}$, Jorgensen S, Pederson K. Microbiological evaluation of jejunal aspirates and faecal samples after oral administration of bifidobacteria and lactic acid bacteria. J Appl Bacteriol 1994; 76: 469-74.

9. Hunter JO, Alun Jones V. Studies on the pathogenesis of irritable bowel syndrome produced by food intolerance. In: Read NW, ed. Irritable bowel syndrome. London: Grune \& Stratton; 1985. p. 185-90.

10. Kennedy TM, Jones RH. The epidemiology of hysterectomy and irritable bowel syndrome in a UK population. Int J Clin Pract 2000; 54: 647-50.

11. Gwee KA, Graham JC, McKendrick MW, Collins SM, Marshall JS, Walters SJ, et al. Psychometric scores and persistence of irritable bowel after infectious diarrhoea. Lancet 1996; 347: 150-3.

12. Rodriguez LAG, Ruigomez A. Increased risk of irritable bowel syndrome after bacterial gastroenteritis: cohort study. Br Med J 1999; 318: 565-6.

13. Gwee KA. Postinfectious irritable bowel syndrome. Curr Treat Options Gastroenterol 2001; 4: 287-91.

14. Hooker KD, dePiro JT. Effect of antimicrobial therapy on bowel flora. Clin Pharm 1988; 7: 878-88.

15. Edlund C, Beyer G, Nord CE. Antimicrobial treatment of peridontal diseases disturbs the human ecology: a review. Scand J Infect Dis Suppl 1996; 8: 331-41.

16. Alun Jones V, Wilson AJ, Hunter JO, Robinson RE. The aetiological role of antibiotic prophylaxis with hysterectomy in irritable bowel syndrome. J Obstet Gynaecol 1984; 5(Suppl 1): S22-3.

17. Mendall MA, Kumar D. Antibiotic use, childhood affluence and irritable bowel syndrome (IBS). Eur J Gastroenterol Hepatol 1998; 10: 59-62.

18. Balsari A, Ceccarelli A, Dubini F, Fesce E, Poli G. The fecal microbial population in the irritable bowel syndrome. Microbiologica 1982; 5: 185-94.

19. Bradley HK, Wyatt GM, Bayliss CE, Hunter JO. Instability in the faecal flora of a patient suffering from food-related irritable bowel syndrome. J Med Microbiol 1987; 23: 29-32.

20. Wyatt GM, Bayliss CE, Lakey AF, Bradley HK, Hunter JO, Alun Jones V. The faecal flora of two patients with food-related irritable bowel syndrome during challenge with symptom-provoking foods. J Med Microbiol 1988; 26: $295-9$. 
21. Madden JAJ, Plummer S, Sen S, Dear K, Tarry S, Hunter JO. Comparison of the caecal and faecal microflora of healthy subjects and patients with irritable bowel syndrome (IBS). Gut 2001; 48(Suppl 1): A58.

22. Macfarlane GT, Cummings JH. Probiotics, infection and immunity. Curr Opin Infect Dis 2002; 15: 1-6.

23. Berg RD. Probiotics, prebiotics or "conbiotics"? Trends Microbiol 1998; 6: 89-93.

24. Tannock GW. Probiotics properties of lactic acid bacteria: plenty of scope for fundamental R\&D. Trends Biotechnol 1997; 15: 270-4.

25. Holzapfel WH, Haberer P, Snel J, Schillinger U, Huis in't Veld JHJ. Overview of gut flora and probiotics. Int J Food Microbiol 1998; 41: 85-101.

26. Shanahan F. Inflammatory bowel disease: immunodiagnostics, immunotherapeutics, and ecotherapeutics. Gastroenterology 2001; 120: 622-35.

27. Niedzielin K, Kordecki H, Kosik R. New possibility in the treatment of irritable bowel syndrome: probiotics as a modification of the flora of the colon. Gastroenterology 1998; 114: A402.

28. Niedzielin K, Kordecki H, Birkenfeld B. A controlled, double-blind, randomized study on the efficacy of Lactobacillus plantarum $299 \mathrm{~V}$ in patients with irritable bowel syndrome. Eur J Gastroenterol Hepatol 2001; 13: 1143-7.

29. Halpern GM, Prindiville T, Blankenburg M, Hsia T, Gershwin ME. Treatment of irritable bowel syndrome with Lacteol Fort: a randomized, double-blind, crossover trial. Am J Gastroenterol 1996; 91: 1579-85.

30. Brigidi P, Vitali B, Swennen E, Bazzocchi G, Matteuzzi D. Effects of probiotic administration upon the composition and enzymatic activity of human fecal microbiota in patients with irritable bowel syndrome or functional diarrhea. Res Microbiol 2001; 152: 735-41.

31. Nobaeck S, Johansson ML, Molin G, Ahrne S, Jeppsson B. Alteration of intestinal microflora is associated with reduction in abdominal bloating and pain in patients with irritable bowel syndrome. Am J Gastroenterol 2000; 95: 1231-8.

32. O'Sullivan MA, O'Morain CA. Bacterial supplementation in the irritable bowel syndrome. A randomised double-blind placebo-controlled crossover study. Dig Dis Sci 2000; 32: 302-4.

33. Bazzocchi G, Gionchetti P, Almerigi PF, Amadini C, Campieri M. Intestinal microflora and oral bacteriother- apy in irritable bowel syndrome. Dig Liver Dis 2002; 34(Suppl 2): S48-53.

34. Gotz VP, Romankiewicz JA, Moss J, Murray HW. Prophylaxis against ampicillin associated diarrhoea with Lactobacillus preparation. Am J Hosp Pharm 1979; 36: 754-7.

35. Surawicz CM, Elmer GW, Speelman P, McFarland LV, Chinn J, Van Belle G. Prevention of antibiotic-associated diarrhoea by Saccharomyces boulardii. Gastroenterology 1989; 96: 981-8.

36. Wunderlich PF, Braun L, Fumagalli I, D’Apuzzo V, Heim F, Karly M, et al. Double-blind report on the efficacy of lactic acid-producing Enterococcus SF68 in the prevention of antibiotic-associated diarrhoea and in the treatment of acute diarrhoea. J Int Med Res 1989; 17: 333-8.

37. McFarland LV, Surawicz CM, Greenberg RN, Elmer GW, Moyer KA, Melcher SA, et al. Prevention of beta lactam-associated diarrhea by Saccharomyces boulardii compared with placebo. Am J Gastroenterol 1995; 90: 439-48.

38. Vanderhoof JA, Whitney DB, Antoson DL, Hanner TL, Lupo JV, Young RJ. Lactobacillus GG in the prevention of antibiotic-associated diarrhoea in children. J Pediatr 1999; 135: 564-8.

39. Sen S, Mullan M, Parker TJ, Tarry SA, Hunter JO. Effects of Lactobacillus plantartum 299v (ProViva) on symptoms and colonic fermentation in patients with IBS. Gut 2001; 48(Suppl 1): A57.

40. Madden JAJ, Plummer S, Sen S, Dear K, Tarry S, Hunter JO. The effects of probiotic supplementation on the caecal biopsy-associated and caecal luminal microflora of patients with irritable bowel syndrome (IBS) treated with antibiotics. Gut 2001;49(Suppl 3): Abstract No. 1907.

\footnotetext{
Jennifer Madden, $\mathrm{PhD}$

Tiernaleague

Carndonagh

Co. Donegal

Ireland

Tel: + 3536433922

Fax: +3536439022

E-mail: jennifermadden@hotmail.com
} 